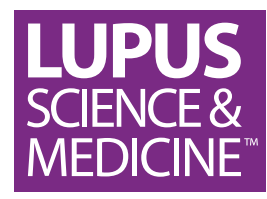

\title{
ANA positivity and complement level in pleural fluid are potential diagnostic markers in discriminating lupus pleuritis from pleural effusion of other aetiologies
} Der-Yuan Chen, ${ }^{1,2,3}$ Yen-Hsiang Huang, ${ }^{4,5}$ Yi-Ming Chen, ${ }^{6,7,8}$ Jeremy J W Chen,
Tsung-Ying Yang,

\section{ABSTRACT}

Objective Lupus pleuritis is the most common pulmonary manifestation of systemic lupus erythematosus (SLE). We aimed to compare various biomarkers in discriminating between pleural effusions due to lupus pleuritis and other aetiologies.

Methods We determined in 59 patients (16 patients with SLE and 43 patients without SLE) pleural fluid levels of high-mobility group box 1 , soluble receptor for advanced glycation end products (SRAGE), adenosine deaminase (ADA), interleukin (IL) 17A, tumour necrosis factor- $\alpha$, antinuclear antibodies (ANA), and complements C3 and C4. Results We found significant differences in the pleural fluid level of sRAGE, ADA, IL-17A, C3 and C4, and in the proportion of ANA positivity, among lupus pleuritis and other groups with pleural effusion. Specifically, ANA positivity (titre $\geq 1: 80$ ) achieved a high sensitivity of $91 \%$, specificity of $83 \%$ and negative predictive value (NPV) of $97 \%$ in discriminating lupus pleuritis from non-lupus pleural effusion. A parallel combination of the level of C3 $(<24 \mathrm{mg} / \mathrm{dL})$ and $\mathrm{C} 4(<3 \mathrm{mg} / \mathrm{dL})$ achieved a sensitivity of $82 \%$, specificity of $89 \%$ and NPV of $93 \%$ in discriminating lupus pleuritis from non-lupus exudative pleural effusion. Conclusions In conclusion, ANA, C3 and C4 in pleural fluid are useful in discriminating lupus pleuritis from pleural effusion due to other aetiologies with high NPV.

\section{INTRODUCTION}

Systemic lupus erythematosus (SLE) is an autoimmune disease that likely leads to serious complications. ${ }^{1}$ Lupus pleuritis is the most common pulmonary manifestation of SLE, with a prevalence of $45 \%-60 \% .^{2}$ It is sometimes even the initial presentation in patients with SLE. ${ }^{3}$ However, there are other causes of pleurisy, such as infections, congestive heart failure and malignancy. ${ }^{4}$ The differential diagnosis of lupus pleuritis is challenging but crucial for early optimal treatment. Analyses of pleural fluid in patients with lupus pleuritis revealed mostly exudative

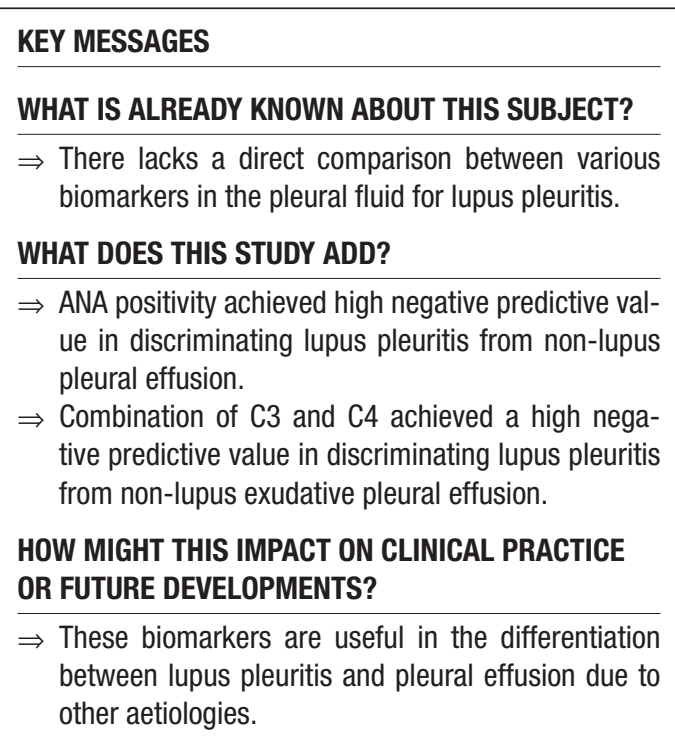

changes, dominated with either neutrophils or lymphocytes, and with decreased levels of complements C3 as well as C4, and the presence of ANA. ${ }^{5}$ Nevertheless, the diagnostic values of various potential biomarkers for lupus pleuritis have not been directly compared in a single study. Furthermore, the distribution of these biomarkers in non-lupus pleural effusion may vary among the different aetiologies, which has not been fully explored in previous studies.

High-mobility group box 1 (HMGB1), a DNA-binding nuclear protein, is an endogenous damage-associated molecular pattern. ${ }^{6}$ HMGB1 promotes an inflammatory response through the receptor for advanced glycation end products (RAGE). ${ }^{7}$ Soluble RAGE (sRAGE) is a truncated form of RAGE and primarily acts as a decoy receptor to capture proinflammatory ligands like HMGB1. ${ }^{8}$ Several studies have demonstrated increased 
circulating levels of HMGB1 and decreased levels of sRAGE in patients with autoimmune diseases such as rheumatoid arthritis (RA) ${ }^{9} 10$ and SLE. ${ }^{11-13}$ Therefore, perturbations in the levels of HMGB1 and sRAGE are postulated to be present in the pleural fluid of these patients.

Herein, we determined the pleural fluid levels of biomarkers potentially useful in discriminating lupus pleuritis from pleural effusion of different aetiologies.

\section{METHODS \\ Patients}

We prospectively enrolled 16 consecutive patients with SLE diagnosed according to the 1997 American College of Rheumatology criteria ${ }^{14}$ presenting with pleural effusion between March 2015 and December 2020. Their median disease duration was 5 years and half of them had lupus nephritis. The disease activity for SLE was evaluated by the Systemic Lupus Erythematosus Disease Activity Index (SLEDAI) ${ }^{15}$ Of the patients, 11 had lupus pleuritis, 4 had fluid overload (3 with nephrotic syndrome and 1 with heart failure) and 1 had malignant pleural effusion (cancer of unknown primary). We also enrolled 43 patients without SLE: 11 with infection-related pleural effusion (5 parapneumonic pleural effusion and 6 empyema), 18 with malignant pleural effusion based on pathological findings (8 lung cancer, 4 gynaecological cancer, 2 breast cancer, 2 gastrointestinal cancer, 1 hepatoma and 1 transitional cell carcinoma of the urinary bladder) and 14 with fluid overload (9 with heart failure and 5 with hepatic hydrothorax). The above diagnoses were made retrospectively at the discretion of the treating physician. In particular, lupus pleuritis was diagnosed based on associated symptoms, exclusion of other possible causes and treatment response. We determined whether a pleural effusion is exudative or transudative based on Light's criteria. ${ }^{16}$ Written consent from each participant was obtained.

\section{Determination of HMGB1 and SRAGE}

The level of HMGB1 in the pleural effusion was determined using an ELISA kit (Chondrex, Redmond, Washington, USA) according to the manufacturer's instructions. In brief, $100 \mu \mathrm{L}$ of the capture antibody were added in each well at $4^{\circ} \mathrm{C}$ overnight. Then we added $50 \mu \mathrm{L}$ pleural fluid sample or protein standards. Then $100 \mu \mathrm{L}$ of the detection antibody were added and incubated at $37^{\circ} \mathrm{C}$. After rinsing, $100 \mu \mathrm{L}$ of streptavidin peroxidase were added and incubated at room temperature for $30 \mathrm{~min}$. Detection was performed with tetramethylbenzidine solution. Optical density was measured at $450 \mathrm{~nm}$ using a DAR800 microplate reader (Cortez Diagnostics, California, USA). One sample from the lupus pleuritis group did not undergo determination of HMGB1. The level of sRAGE in the pleural effusion was determined using an ELISA kit (Quantikine, R\&D Systems, Abingdon, UK) in accordance with the manufacturer's instructions. Briefly, we added $100 \mu \mathrm{L}$ of diluent to each well. We then added $50 \mu \mathrm{L}$ of the patient's pleural fluid per well and incubated at room temperature for 2 hours. After rinsing, we added $200 \mu \mathrm{L}$ of detection antibody conjugated to horseradish peroxidase and incubated at room temperature for 2 hours. Detection was performed with tetramethylbenzidine solution. Optical density was measured at $450 \mathrm{~nm}$ using Thermo Multiskan EX Microplate Photometer (Thermo Fisher Scientific). For each sample, duplicate measurements were made to obtain an average value.

\section{Measurement of IL-17A and TNF- $\alpha$ levels}

The pleural fluid level of two proinflammatory cytokines, interleukin 17-A (IL-17A) and tumour necrosis factor- $\alpha$ (TNF- $\alpha$ ), was determined with ELISA (Quantikine) and chemiluminescent ELISA (QuantiGlo, R\&D Systems), respectively. Optical densities were measured at $450 \mathrm{~nm}$ for IL-17A using Thermo Multiskan EX Microplate Photometer (Thermo Fisher Scientific) and the luminescence of TNF- $\alpha$ was determined using Beckman Coulter DTX 880 Multimode Detector (Beckman Coulter).

\section{Determination of pleural fluid levels of ANA, C3, C4 and ADA}

The pleural fluid level of ANA was determined with indirect immunofluorescence using a Hep-2 cell line (Medical \& Biological Laboratories, Nagoya, Japan). The level of C3 and C4 was measured with immunoturbidimetry (Siemens Healthcare Diagnostics, Tarrytown, New York, USA). Adenosine deaminase (ADA) activity was evaluated before June 2016 using the endpoint method (Denka Seiken, Japan), ${ }^{17}$ and after June 2016 using the enzymatic method (InnoChem, Pyeongtaek, South Korea). ${ }^{18}$ Values obtained with these two methods were harmonised using Passing-Bablok regression. ${ }^{19}{ }^{20}$ One patient without SLE and with malignant pleural effusion did not undergo C3 level determination. One patient without SLE and with malignant pleural effusion, and one without SLE and with fluid overload did not undergo C4 level examination. One patient without SLE and with infection-related pleural effusion did not undergo ADA activity examination.

\section{Statistical analyses}

Statistical analyses were performed using Stata V.15.0 software. Quantitative data were presented as median and IQR unless specified otherwise. Kruskal-Wallis test and $\chi^{2}$ test were performed to assess differences between patients with lupus pleuritis and patients without SLE and with pleural effusion due to other aetiologies. For between-group comparisons of numerical variables (lupus pleuritis vs infection-related pleural effusion, malignant pleural effusion and fluid overload), non-parametric Mann-Whitney $\mathrm{U}$ test and $\chi^{2}$ test were used. Bonferroni's correction was undertaken for multiple comparisons. The diagnostic performance for lupus pleuritis of each biomarker and their combinations was determined. The area under the receiver operating characteristic curve (AUC) was calculated using MedCalc statistical software 
Table 1 Baseline characteristics

\begin{tabular}{|c|c|c|c|c|}
\hline & $\begin{array}{l}\text { Lupus pleuritis } \\
(n=11)\end{array}$ & $\begin{array}{l}\text { Infection-related pleural } \\
\text { effusion }(n=11)\end{array}$ & $\begin{array}{l}\text { Malignant pleural } \\
\text { effusion }(n=18)\end{array}$ & Fluid overload $(n=14)$ \\
\hline \multicolumn{5}{|l|}{ Demographics } \\
\hline Female sex $(\%)^{\star}$ & $9(82)$ & $2(18) \dagger$ & $10(56)$ & $3(21) \dagger$ \\
\hline \multicolumn{5}{|l|}{ Pleural effusion } \\
\hline Neutrophils (\%)* & $8(3-27)$ & $81(35-94) \dagger$ & $6(0-17)$ & $5(2-11)$ \\
\hline Lymphocytes (\%)* & $24(10-52)$ & $9(5-27)$ & $38.5(21-74)$ & $49(38-62)$ \\
\hline Protein $(\mathrm{mg} / \mathrm{dL})^{\star}$ & $3.7(2.2-4.5)$ & $4.1(2.9-4.9)$ & $3.7(3.3-4.2)$ & $1.8(1.4-2.8) \dagger$ \\
\hline $\mathrm{LDH}(\mathrm{U} / \mathrm{L})^{*}$ & 125 (98-203) & 1081 (403-1935)† & $208(155-394)$ & $79(63-96) \dagger$ \\
\hline \multicolumn{5}{|c|}{$\begin{array}{l}\text { Data are presented as median (IQR) or number (percentage). } \\
{ }^{*} \mathrm{P}<0.05 \text { as determined by Kruskal-Wallis test or } \chi^{2} \text { test. } \\
+\mathrm{P}<0.016 \text { versus lupus pleuritis as determined by Mann-Whitney } U \text { test or } \chi^{2} \text { test based on adjustment for multiple comparisons. } \\
\mathrm{LDH} \text {, lactate dehydrogenase. }\end{array}$} \\
\hline
\end{tabular}

(V.9.3; MedCalc Software, Ostend, Belgium). Youden index was calculated to set the optimal cut-off point. We also used Mann-Whitney $\mathrm{U}$ test to compare between patients with lupus pleuritis and SLE patients with fluid overload. Statistical significance was set at a two-sided $\mathrm{p}$ value of $<0.05$.

\section{RESULTS}

Demographic data and clinical characteristics of patients with lupus pleuritis and patients without SLE and with pleural effusion of other aetiologies

Patients with infection-related pleural effusion were the oldest, with a median age of 71 years, whereas patients with lupus pleuritis were the youngest, with a median age of 28 years (table 1). The group of patients with lupus pleuritis was predominantly female $(82 \%)$, whereas the group of patients with infection-related pleural effusion was predominantly male $(82 \%)$. Patients with infectionrelated pleural effusion had the highest white cell count, the highest percentage of neutrophils, as well as the highest level of lactate dehydrogenase in the pleural fluid.

Levels of HMGB1, sRAGE, ADA, IL-17A, TNF- $\alpha$, ANA, C3 and C4 in the pleural fluid from patients with lupus pleuritis and patients without SLE and with pleural effusion of other aetiologies

We found significant differences in the level of sRAGE, ADA activity, IL-17A, C3 and C4, and in the proportion of ANA positivity, among the different groups with pleural effusion (table 2 and figure 1). The proportion of ANA positivity was higher whereas the level of C4 was lower in the lupus pleuritis group when compared with the infection-related pleural effusion group. There were lower levels of C3 and a trend towards lower ADA activity and levels of IL-17A in the lupus pleuritis group when compared with the infection-related pleural effusion group. The proportion of ANA positivity was higher whereas the level of C4 was lower in the lupus pleuritis group when compared with the malignant pleural effusion group. There appeared a trend towards higher levels of sRAGE $(p=0.059)$ but lower levels of C3 in the lupus pleuritis group when compared with the malignant pleural effusion group. The proportion of ANA positivity was higher and the level of C3 tended to be higher $(p=0.080)$ in the lupus pleuritis group than the fluid overload-related pleural effusion group. The most frequent ANA pattern was fine speckled (90\%) in lupus pleuritis, which was not different from pleural effusion due to other aetiologies.

Diagnostic performance of HMGB1, sRAGE, ADA, IL-17A, TNF- $\alpha$, ANA, C3 and C4 in the pleural fluid to discriminate Iupus pleuritis from pleural effusion of other aetiologies Diagnostic performance regarding pleural fluid levels of HMGB1, sRAGE, ADA activity, IL-17A, TNF- $\alpha$, C3 and C4 is illustrated in table 3 and figure 2. C3 and C4 levels both had good diagnostic performance in differentiating between lupus nephritis and infection-related pleural effusion (AUC: 0.81 and 0.82, respectively). C4 levels also had good diagnostic performance in differentiating between lupus pleuritis and malignant pleural effusion (AUC: 0.83 ). The sensitivity and specificity of ANA and C3/C4 at different cut-off points and their combinations are shown in table 3 and online supplemental table S1. The sensitivity and specificity of ANA were both good in differentiating between lupus pleuritis and pleural effusion of other aetiologies. A parallel combination of C3 $(<24 \mathrm{mg} / \mathrm{dL})$ and $\mathrm{C} 4(<3 \mathrm{mg} / \mathrm{dL})$ had good diagnostic 
Table 2 Level of potential biomarkers in the pleural fluid

\begin{tabular}{|c|c|c|c|c|}
\hline Potential markers & $\begin{array}{l}\text { Lupus pleuritis } \\
(n=11)\end{array}$ & $\begin{array}{l}\text { Infection-related pleural } \\
\text { effusion }(n=11)\end{array}$ & $\begin{array}{l}\text { Malignant pleural } \\
\text { effusion }(n=18)\end{array}$ & $\begin{array}{l}\text { Fluid overload } \\
(n=14)\end{array}$ \\
\hline \multicolumn{5}{|l|}{ Immunological markers } \\
\hline HMGB1 (ng/mL) & $0.48(0.39-18.02)$ & $4.24(0.48-30.5)$ & $1.03(0.62-2.53)$ & $0.54(0.43-0.77)$ \\
\hline sRAGE $(p g / m L)^{*}$ & $4232(1256-5096)$ & $3030(315-4721)$ & 3020 (1900-3806) & 4496 (3915-4956) \\
\hline $\mathrm{ADA}(\mathrm{U} / \mathrm{L})^{*}$ & $13(7-28)$ & $37(19-87)$ & $12(10-20)$ & $8(6-11)$ \\
\hline \multicolumn{5}{|l|}{ Proinflammatory cytokines } \\
\hline IL-17A $(\mathrm{pg} / \mathrm{mL})^{\star}$ & 3.07 (1.54-3.74) & $4.46(2.4-15.33)$ & $2.31(1.79-2.84)$ & $3.43(1.79-6.81)$ \\
\hline TNF- $\alpha(p g / m L)$ & $5.99(2.21-21.56)$ & $5.93(4.31-20.69)$ & $7.08(4.89-8.65)$ & $4.37(2.91-8.09)$ \\
\hline \multicolumn{5}{|l|}{ SLE-related markers } \\
\hline ANA titre $\geq 1: 80, \mathrm{n}(\%)^{\star}$ & $10(91)$ & $2(18) \dagger$ & $4(24) \dagger$ & $1(7) \dagger$ \\
\hline \multicolumn{5}{|l|}{ Pattern } \\
\hline Fine speckled & $9(90)$ & $2(100)$ & $2(50)$ & $1(100)$ \\
\hline Homogenous & $6(60)$ & $1(50)$ & $1(25)$ & $1(100)$ \\
\hline Coarse speckled & $3(30)$ & $0(0)$ & $0(0)$ & $0(0)$ \\
\hline ANA titre $\geq 1: 160, n(\%)^{*}$ & $9(82)$ & $2(18) \dagger$ & $2(12) \dagger$ & $0(0) \dagger$ \\
\hline ANA titre $\geq 1: 320, n(\%)^{*}$ & $8(73)$ & $1(9) \dagger$ & $1(6) \dagger$ & $0(0) \dagger$ \\
\hline $\mathrm{C} 3(\mathrm{mg} / \mathrm{dL})^{\star}$ & $22.3(17.1-40.0)$ & $44.2(24.1-78.8) \dagger$ & $38.4(33.2-53.0)$ & $14.0(5.5-25.3)$ \\
\hline Protein-adjusted $\mathrm{C} 3^{\star} \ddagger$ & $6.56(3.82-11.36)$ & $13.00(8.31-18.7)$ & $11.15(9.50-12.41)$ & $7.49(6.11-9.47)$ \\
\hline $\mathrm{C} 4(\mathrm{mg} / \mathrm{dL})^{*}$ & $2.5(0.6-6.0)$ & $8.5(6.1-13.9) \dagger$ & $8.3(6.4-10.0) \dagger$ & $3.4(1.2-4.7)$ \\
\hline Protein-adjusted $\mathrm{C} 4^{*} \ddagger$ & $0.73(0.16-2.35)$ & $2.83(1.27-4.27) \dagger$ & $2.27(1.64-3.14) \dagger$ & $1.53(0.88-2.07)$ \\
\hline
\end{tabular}

Data are presented as median (IQR) or number (percentage).

${ }^{*} \mathrm{P}<0.05$ as determined by Kruskal-Wallis test or $\chi^{2}$ test.

$\dagger \mathrm{P}<0.016$ versus lupus pleuritis as determined by Mann-Whitney $U$ test or $\chi^{2}$ test based on adjustment for multiple comparisons.

‡C3 or C4 levels divided by pleural fluid levels of protein and then multiplied by 1000 .

ADA, adenosine deaminase; HMGB1, high-mobility group box 1; IL-17A, interleukin 17A; sRAGE, soluble receptor for advanced glycation end products; TNF- $\alpha$, tumour necrosis factor- $\alpha$.

performance in differentiating lupus pleuritis versus infection-related pleural effusion and malignant pleural effusion.

Levels of HMGB1, sRAGE, proinflammatory cytokines and potential biomarkers in the pleural fluid between lupus pleuritis and pleural effusion of other aetiologies in patients with SLE

As shown in online supplemental table S2, nine (56\%) patients with SLE had an active disease (SLEDAI $\geqq 4$ ). The fluid overload group had a higher proportion of lupus nephritis and nephrotic range proteinuria than the lupus pleuritis group. We demonstrated levels of HMGB1, sRAGE, proinflammatory cytokines and potential biomarkers, including pleural effusion/serum ANA, C3 and C4 ratios, ${ }^{21}{ }^{22}$ between lupus pleuritis and pleural effusion of other aetiologies in patients with SLE. We only found a trend towards higher C3 levels in lupus pleuritis than fluid overload-related pleural effusion in patients with SLE (online supplemental figure S1).

\section{DISCUSSION}

Pleural effusion is a common manifestation of SLE. ${ }^{23}$ Given multiple causes of pleural effusion, it is useful to identify biomarkers for discriminating lupus pleuritis from pleural effusion of other aetiologies. We found in the present study that pleural fluid levels of ANA, C3 and C4 are potentially useful in discriminating lupus pleuritis from pleural effusion of other aetiologies.

Circulating levels of HMGB1 were elevated in RA and SLE. ${ }^{9}{ }^{11}{ }^{23}$ Upregulated HMGB1 is also present in patients with infectious diseases. Several studies have reported markedly elevated serum levels of HMGB1 in patients with sepsis ${ }^{24}$ or severe sepsis ${ }^{25}$ and a positive correlation between plasma levels of HMGB1 and organ dysfunction in septic shock. ${ }^{26}$ A prior study also reported elevated levels of HMGB1 in malignant and inflammatory pleural effusion compared with transudative pleural effusion. ${ }^{27}$ Although the median level of HMGB1 appeared higher in infection-related pleural effusion than lupus pleuritis in the present study, the elevated level was not statistically significant. sRAGE acts as a suppressor of the inflammatory response in the RAGE axis. Circulating levels of sRAGE were decreased in patients with RA and SLE. ${ }^{10} 121328$ A previous study reported lower levels of pleural fluid sRAGE in patients with bacterial pneumonia compared with those with tuberculosis or lung cancer. ${ }^{29}$ We only observed a trend of higher levels of pleural fluid 
A HMGB1

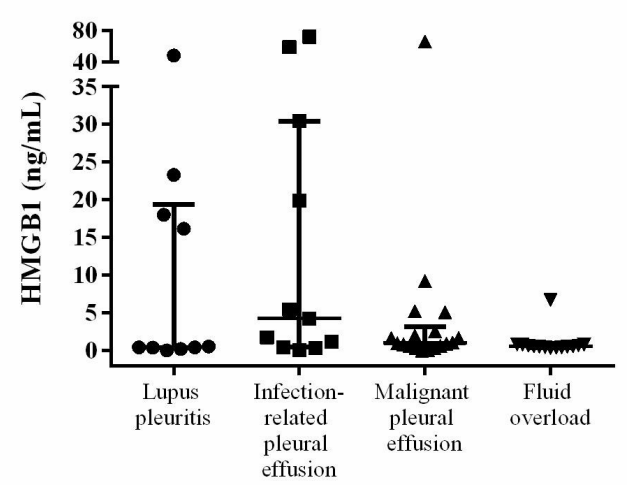

C ADA activity

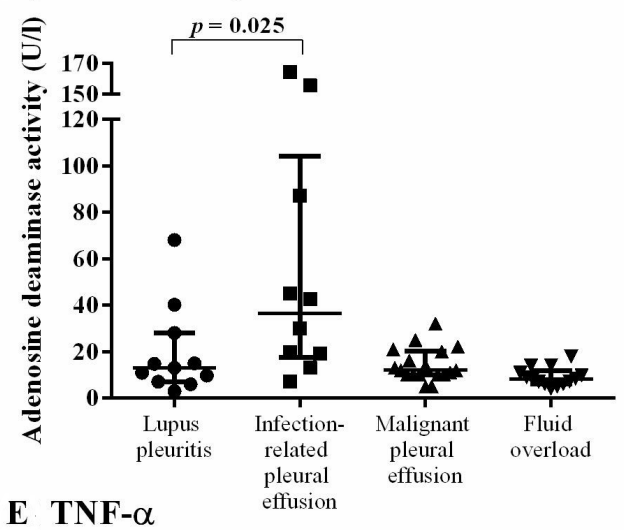

E TNF- $\alpha$

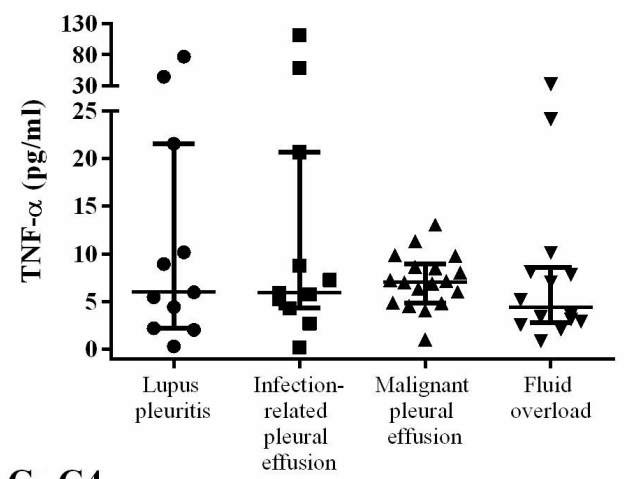

G C4

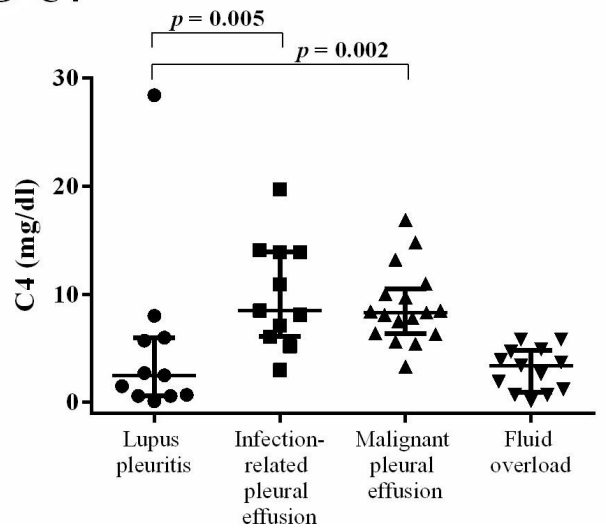

\section{B SRAGE}

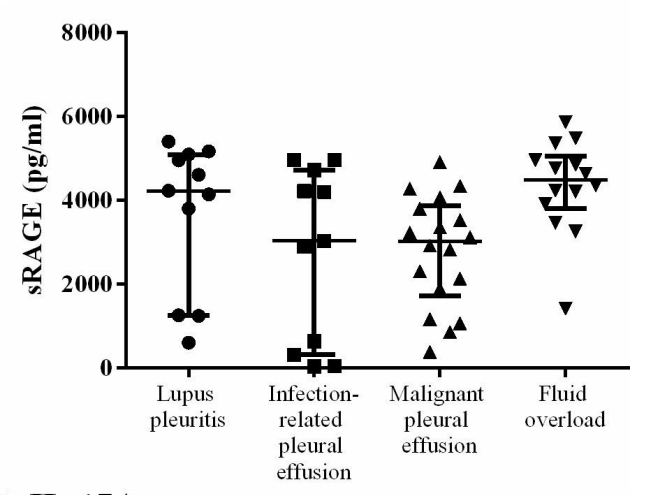

D IL-17A

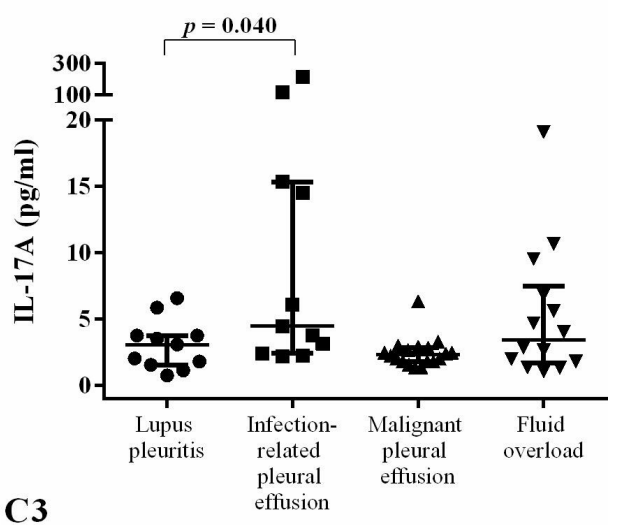

C3

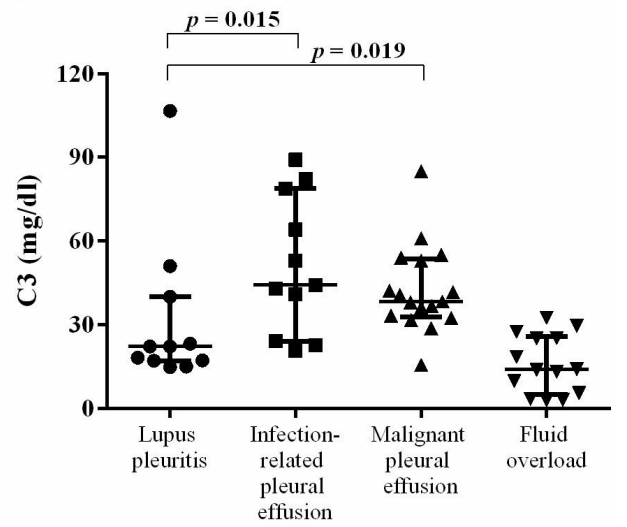

ADA catalyses the deamination of adenosine, which is a crucial suppressor of the inflammation. ${ }^{30}$ Moreover,
sRAGE with lupus pleuritis when compared with malignant pleural effusion. 
Table 3 Diagnostic performance of potential biomarkers

$\begin{array}{ll}\begin{array}{l}\text { Lupus pleuritis vs infection- } \\ \text { related pleural effusion }\end{array} \quad \text { malignant pleural effusion } & \text { Lupus pleuritis vs fluid overload }\end{array}$

AUC $(95 \% \mathrm{Cl})$ for numerical variables

Immunological markers

$\begin{array}{llll}\text { HMGB1 } & 0.62(0.38 \text { to } 0.82) & 0.53(0.33 \text { to } 0.72) & 0.55 \text { (0.34 to } 0.75) \\ \text { sRAGE } & 0.67(0.44 \text { to } 0.85) & 0.71(0.52 \text { to } 0.86) & 0.58 \text { (0.37 to } 0.78) \\ \text { ADA activity } & 0.72(0.55 \text { to } 0.93) & 0.52(0.33 \text { to } 0.71) & 0.70 \text { (0.48 to } 0.86)\end{array}$

Proinflammatory cytokines

$\begin{array}{llll}\text { IL-17A } & 0.70(0.47 \text { to } 0.87) & 0.63(0.43 \text { to } 0.80) & 0.59(0.38 \text { to } 0.78) \\ \text { TNF- } \alpha & 0.53(0.31 \text { to } 0.74) & 0.52(0.33 \text { to } 0.71) & 0.57(0.36 \text { to } 0.73)\end{array}$

SLE-related markers

$\begin{array}{llll}\text { C3 } & 0.81(0.58 \text { to } 0.94) & 0.77(0.57 \text { to } 0.90) & 0.71(0.49 \text { to } 0.87) \\ \text { C4 } & 0.82(0.60 \text { to } 0.95) & 0.83(0.64 \text { to } 0.94) & 0.52 \text { (0.31 to } 0.73)\end{array}$

Sensitivity/specificity for binary variables

\begin{tabular}{llll} 
ANA titre $\geq 1: 80, \%$ & $91 / 82$ & $91 / 76$ & $91 / 93$ \\
\hline ANA titre $\geq 1: 160, \%$ & $82 / 82$ & $82 / 88$ & $82 / 100$ \\
ANA titre $\geq 1: 320, \%$ & $73 / 91$ & $73 / 94$ & $73 / 100$
\end{tabular}

Data are presented as AUC $(95 \% \mathrm{Cl})$.

ADA, adenosine deaminase; AUC, area under the receiver operating characteristic curve; HMGB1, high-mobility group box 1; IL-17A, interleukin 17A; sRAGE, soluble receptor for advanced glycation end products; TNF- $\alpha$, tumour necrosis factor- $\alpha$.

ADA is involved in the differentiation and maturation of the immune cells such as lymphocytes. ${ }^{31}$ Elevated serum ADA activity was found in patients with SLE. ${ }^{31}{ }^{32} \mathrm{~A}$ previous study reported elevated ADA activities in tuberculous pleurisy compared with lupus pleuritis. Similarly, we observed a trend towards higher ADA activity in infection-related pleural effusion compared with lupus pleuritis. Taken together, ADA activity in the pleural fluid was upregulated in infection-related pleural effusion. Regarding pleural fluid cytokines, we observed a trend of a higher level of IL-17A in infection-related pleural effusion compared with lupus pleuritis. This finding is in line with its known role in bacterial infection. ${ }^{33}$

The significant biomarkers for lupus pleuritis included the higher proportion of ANA positivity and lower levels of C4. Notably, ANA positivity achieved a high sensitivity of $91 \%$, a specificity of $83 \%$, a positive predictive value (PPV) of $59 \%$ and a high negative predictive value (NPV) of $97 \%$ in discriminating lupus pleuritis from pleural effusion of all other aetiologies combined (data not shown).

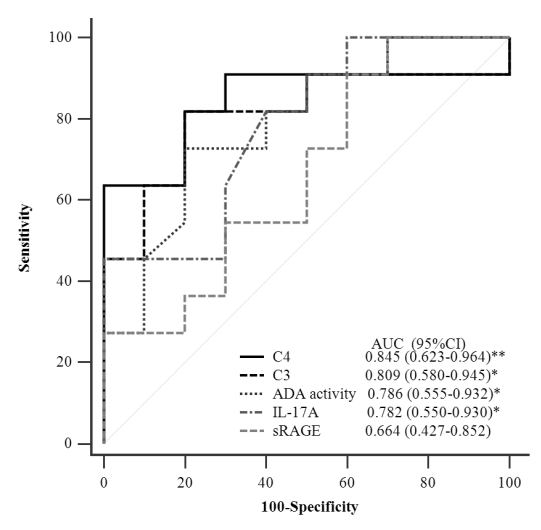

(a) Lupus pleuritis vs Infection-related pleural effusion

Figure 2 Receiver operating characteristic curve for pleural fluid level of potential biomarkers with respect to (a) lupus pleuritis vs. infection-related pleural effusion, (b) lupus pleuritis vs. malignant pleural effusion, and (c) lupus pleuritis vs. fluid overload. One patient without SLE with malignant pleural effusion did not undergo C3 level determination. One patient without SLE and with malignant pleural effusion, and one without SLE and with fluid overload did not undergo C4 level examination. One patient without SLE and with infection-related pleural effusion did not undergo ADA activity examination. ADA, adenosine deaminase; AUC, area under the receiver operating characteristic curve; IL-17A, interleukin 17A; sRAGE, soluble receptor for advanced glycation end products.
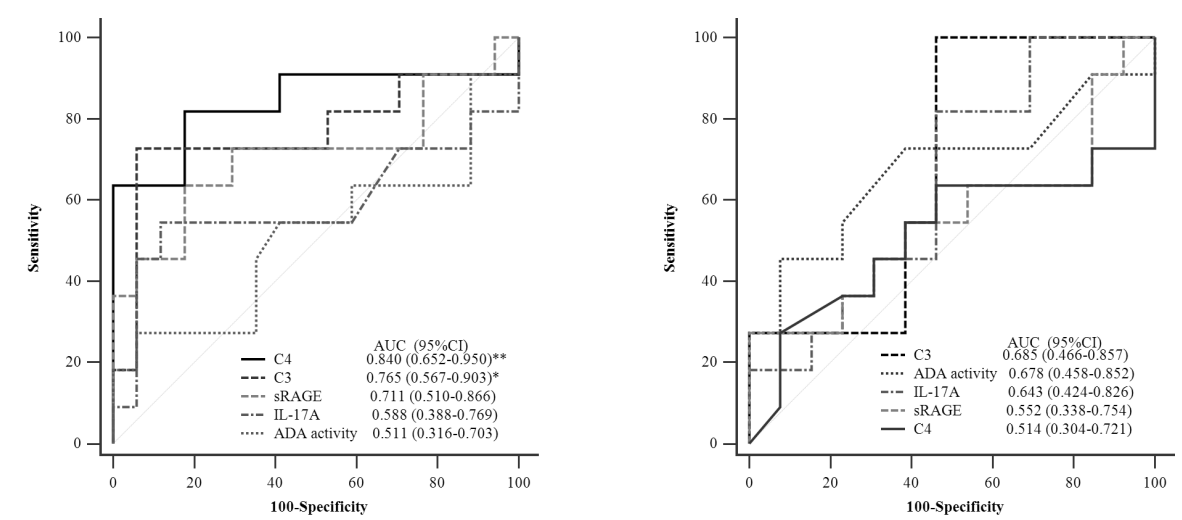

(c) Lupus pleuritis vs Fluid overlond 
Our results are consistent with previous reports on high titre ANA ( $\geqq 1: 160)$ in pleural fluid being a sensitive but less specific indicator of lupus pleuritis, ${ }^{21} 223435$ although we found the positivity for a low titre ANA ( $\geqq 1: 80$ ) having a better diagnostic performance over the other titre thresholds. Resonated with our findings, the newly updated diagnostic criteria for SLE have taken a low titre ANA ( $\geqq 1: 80)$ as the entry criterion to improve its sensitivity ${ }^{3637}$ To be noted, $89 \%$ of patients with lupus pleuritis had pleural fluid/serum ANA ratio $\leqq 1$ (data not shown), which implied the origin of pleural fluid ANA being the circulating blood.

In agreement with previous studies, ${ }^{38-41}$ we revealed lower pleural fluid levels of C3 and C4 in patients with lupus pleuritis. Our results showed that either C3 or C4 level had good diagnostic performance in discriminating lupus pleuritis from infection-related or malignant pleural effusion. Furthermore, a parallel combination of C3 $(<24 \mathrm{mg} / \mathrm{dL})$ and C4 $(<3 \mathrm{mg} / \mathrm{dL})$ showed better diagnostic performance than either biomarker alone. This combination achieved a sensitivity of $82 \%$, a specificity of $89 \%$, a PPV of $75 \%$ and a high NPV of $93 \%$ in differentiating between lupus pleuritis and exudative pleural effusion (infection-related and malignant pleural effusion combined; data not shown). To be noted, the lupus pleuritis group had lower pleural fluid levels of $\mathrm{C} 4$ when compared with infection-related and malignant pleural effusion. This may be partly explained by the concomitant genetic deficiency of C4 in these patients with SLE. ${ }^{42}$ On the contrary, we demonstrated higher pleural fluid levels of C3 in lupus pleuritis when compared with fluid overload-related pleural effusion in patients both without and with SLE. The finding differed from earlier studies which had reported lower C3 levels in lupus pleuritis. ${ }^{38-41}$ However, most of these studies recruited few patients with SLE $(<10)$, and fluid overload-related pleural effusion was under-represented in the control group. Besides, another study reported lower C4 levels in pleural effusion due to heart failure when compared with parapneumonic and malignant pleural effusion. ${ }^{41}$ Notably, we found lower serum levels of C3 in patients with SLE with fluid overload than the lupus pleuritis group (44.25 (IQR 33.05-61.25) $\mathrm{mg} / \mathrm{dL}$ vs 89.8 (IQR 62.2-119) mg/dL; data not shown), which might be the result of a higher proportion of nephrotic range proteinuria in the fluid overload group. This partly explains the lower pleural fluid C3 levels in our patients with SLE with fluid overload. It was also likely that the complement components in the blood had entered the affected tissue (eg, the pleural space) only under inflammation like in exudative pleural effusion.

In our 11 patients with lupus pleuritis, only $1(10 \%)$ had pleural fluid/serum C3 and C4 ratios $>1$. In addition, as demonstrated in table 2, pleural fluid levels of C3 and $\mathrm{C} 4$ adjusted by protein levels appeared lower in the lupus pleuritis group. Furthermore, most $(78 \%)$ of them had lower protein-adjusted pleural fluid levels of C3 and C4 when compared with protein-adjusted serum levels of C3 and C4 (data not shown). These observations are in line with the results of previous studies which suggested activation of the complement cascade locally in lupus pleuritis. ${ }^{38} 4041$ Interestingly, we found a significantly lower pleural fluid levels of C3 (14.2 (IQR 9.9-25.1) $\mathrm{mg} / \mathrm{dL}$ vs 40.7 (IQR 24.1-53.0) $\mathrm{mg} / \mathrm{dL}$ ) and C4 (2.85 (IQR 0.95-4.80) $\mathrm{mg} / \mathrm{dL}$ vs 8.0 (IQR 5.4-10.9) $\mathrm{mg} / \mathrm{dL}$ ) between exudative and transudative pleural effusion in our 59 patients (both $\mathrm{p}<0.001$; data not shown). Their diagnostic performance in comparison with traditional Light's criteria should be explored in the following studies.

There are some limitations to our study. First, our study is limited by the small number of patients with pleural effusion. Patients with lupus pleuritis are difficult to recruit owing to the few number of these cases in clinical practice. A larger multicentre study is required to validate our findings on biomarkers for lupus pleuritis. Nevertheless, we have recruited patients with pleural effusion due to different common aetiologies. We have also comprehensively analysed an array of potential biomarkers in these pleural fluid samples. Second, we did not recruit enough patients with SLE presenting with pleural effusion of other aetiologies. Therefore, our findings cannot be extrapolated to differentiation between autoimmune pleuritis and pleural effusion of other aetiologies in patients with SLE.

\section{CONCLUSIONS}

Our results showed that ANA positivity and levels of C3 and C4 in the pleural fluid could help discriminate lupus pleuritis from pleural effusion of other aetiologies with a high NPV. If we analysed exudative pleural effusion only (exudative lupus pleuritis vs infection-related or malignant pleural effusion; online supplemental table S3), the diagnostic performance of ANA would be slightly better, whereas those of C3 and C4 would not change. We have proposed a diagnostic algorithm (online supplemental figure S2). More studies are needed to validate our findings.

\section{Author affiliations}

${ }^{1}$ Translational Medicine Laboratory, China Medical University Hospital, Taichung, Taiwan

${ }^{2}$ Rheumatology and Immunology Center, China Medical University Hospital, Taichung, Taiwan

${ }^{3}$ College of Medicine, China Medical University, Taichung, Taiwan

${ }^{4}$ Division of Chest Medicine, Taichung Veterans General Hospital, Taichung, Taiwan ${ }^{5}$ Institute of Biomedical Sciences, National Chung Hsing University, Taichung, Taiwan ${ }^{6}$ Department of Medical Research, Taichung Veterans General Hospital, Taichung, Taiwan

${ }^{7}$ Faculty of Medicine, National Yang Ming Chiao Tung University, Hsinchu, Taiwan ${ }^{8} \mathrm{Ph}$.D. Program in Translational Medicine and Rong Hsing Research Center for Translational Medicine, National Chung Hsing University, Taichung, Taiwan ${ }^{9}$ Division of Pulmonary Medicine, Chung Shan Medical University Hospital, Taichung, Taiwan

${ }^{10}$ Institute of Medicine, Chung Shan Medical University, Taichung, Taiwan

${ }^{11}$ School of Medicine, Chung Shan Medical University, Taichung, Taiwan

${ }^{12}$ Division of Allergy, Immunology and Rheumatology, Taichung Veterans General Hospital, Taichung, Taiwan 
Acknowledgements The authors sincerely appreciate the assistance of the Centre for Translational Medicine and the Biostatistics Task Force of Taichung Veterans General Hospital, Taichung, Taiwan.

Contributors D-YC and K-TT had the idea for and designed the study. K-TT and Y$\mathrm{HH}$ drafted the article. K-TT and Y-MC conducted the experiments. JJWC, T-YY and G-CC acquired clinical data and performed data analysis. All the authors critically revised the manuscript for important intellectual content and gave final approval for the version to be published. All the authors agree to be accountable for all aspects of the work in ensuring that questions related to the accuracy or integrity of any part of the work are appropriately investigated and resolved. K-TT is responsible for the overall content as guarantor.

Funding This research was supported by Taichung Veterans General Hospital (TCVGH-1043803B)

Disclaimer The funding source had no role in the design, execution, analyses and interpretation of data in the study.

Competing interests None declared.

Patient consent for publication Not required.

Ethics approval The Institutional Review Board of Taichung Veterans General Hospital approved this study (IRB no CF15024A and CF19263B). Consent was obtained according to the Declaration of Helsinki.

Provenance and peer review Not commissioned; externally peer reviewed. Data availability statement Data are available upon reasonable request. Data are available from the corresponding author upon reasonable request.

Supplemental material This content has been supplied by the author(s). It has not been vetted by BMJ Publishing Group Limited (BMJ) and may not have been peer-reviewed. Any opinions or recommendations discussed are solely those of the author(s) and are not endorsed by BMJ. BMJ disclaims all liability and responsibility arising from any reliance placed on the content. Where the content includes any translated material, BMJ does not warrant the accuracy and reliability of the translations (including but not limited to local regulations, clinical guidelines, terminology, drug names and drug dosages), and is not responsible for any error and/or omissions arising from translation and adaptation or otherwise.

Open access This is an open access article distributed in accordance with the Creative Commons Attribution Non Commercial (CC BY-NC 4.0) license, which permits others to distribute, remix, adapt, build upon this work non-commercially, and license their derivative works on different terms, provided the original work is properly cited, appropriate credit is given, any changes made indicated, and the use is non-commercial. See: http://creativecommons.org/licenses/by-nc/4.0/.

\section{ORCID iD}

Kuo-Tung Tang http://orcid.org/0000-0002-5468-1329

\section{REFERENCES}

1 Gupta S, Kaplan MJ. Bite of the wolf: innate immune responses propagate autoimmunity in lupus. J Clin Invest 2021;131. doi:10.1172/JCl144918. [Epub ahead of print: 01 Feb 2021].

2 Keane MP, Lynch JP. Pleuropulmonary manifestations of systemic lupus erythematosus. Thorax 2000;55:159-66.

3 So C, Imai R, Tomishima Y, et al. Bilateral pleuritis as the initial symptom of systemic lupus erythematosus: a case series and literature review. Intern Med 2019;58:1617-20.

4 Pego-Reigosa JM, Medeiros DA, Isenberg DA. Respiratory manifestations of systemic lupus erythematosus: old and new concepts. Best Pract Res Clin Rheumatol 2009;23:469-80.

5 Wang D-Y. Diagnosis and management of lupus pleuritis. Curr Opin Pulm Med 2002;8:312-6.

6 Scaffidi P, Misteli T, Bianchi ME. Release of chromatin protein HMGB1 by necrotic cells triggers inflammation. Nature 2002;418:191-5.

7 Bianchi ME, Manfredi AA. High-mobility group box 1 (HMGB1) protein at the crossroads between innate and adaptive immunity. Immunol Rev 2007;220:35-46.

8 Maillard-Lefebvre H, Boulanger E, Daroux M, et al. Soluble receptor for advanced glycation end products: a new biomarker in diagnosis and prognosis of chronic inflammatory diseases. Rheumatology 2009;48:1190-6.

9 Andersson U, Erlandsson-Harris $\mathrm{H}$. Hmgb1 is a potent trigger of arthritis. J Intern Med 2004;255:344-50.

10 Pullerits R, Bokarewa M, Dahlberg L, et al. Decreased levels of soluble receptor for advanced glycation end products in patients with rheumatoid arthritis indicating deficient inflammatory control. Arthritis Res Ther 2005;7:R817-24.

11 Abdulahad DA, Westra J, Bijzet J, et al. High mobility group box 1 (HMGB1) and anti-HMGB1 antibodies and their relation to disease characteristics in systemic lupus erythematosus. Arthritis Res Ther 2011;13:R71.

12 Ma C-Y, Ma J-L, Jiao Y-L, et al. The plasma level of soluble receptor for advanced glycation end products is decreased in patients with systemic lupus erythematosus. Scand J Immunol 2012;75:614-22.

13 Yu SL, Wong CK, Szeto CC, et al. Members of the receptor for advanced glycation end products axis as potential therapeutic targets in patients with lupus nephritis. Lupus 2015;24:675-86.

14 Hochberg MC. Updating the American College of rheumatology revised criteria for the classification of systemic lupus erythematosus. Arthritis Rheum 1997;40:40

15 Petri M, Kim MY, Kalunian KC, et al. Combined oral contraceptives in women with systemic lupus erythematosus. $N$ Engl J Med 2005;353:2550-8.

16 Light RW. Clinical practice. pleural effusion. N Engl J Med 2002;346:1971-7.

17 Slaats EH, Asberg EG, van Keimpema AR, et al. A continuous method for the estimation of adenosine deaminase catalytic concentration in pleural effusions with a Hitachi 705 discrete analyser. J Clin Chem Clin Biochem 1985;23:677-82.

18 Delacour H, Bousquet A, Fontan E, et al. Ammonia does not interfere with the Diazyme adenosine deaminase test. Clin Chem Lab Med 2013;51:e225-6.

19 Passing $\mathrm{H}$, Bablok. A new biometrical procedure for testing the equality of measurements from two different analytical methods. Application of linear regression procedures for method comparison studies in clinical chemistry, part I. J Clin Chem Clin Biochem 1983;21:709-20.

20 Müller A, Scholz M, Blankenstein O, et al. Harmonization of growth hormone measurements with different immunoassays by data adjustment. Clin Chem Lab Med 2011;49:1135-42.

21 Good JT, King TE, Antony VB, et al. Lupus pleuritis. clinical features and pleural fluid characteristics with special reference to pleural fluid antinuclear antibodies. Chest 1983;84:714-8.

22 Choi BY, Yoon MJ, Shin K, et al. Characteristics of pleural effusions in systemic lupus erythematosus: differential diagnosis of lupus pleuritis. Lupus 2015;24:321-6.

23 Shi Y, Sandoghchian Shotorbani S, Su Z, et al. Enhanced HMGB1 expression may contribute to Th17 cells activation in rheumatoid arthritis. Clin Dev Immunol 2012;2012:1-8.

24 Sundén-Cullberg J, Norrby-Teglund A, Rouhiainen A, et al. Persistent elevation of high mobility group box-1 protein (HMGB1) in patients with severe sepsis and septic shock. Crit Care Med 2005;33:564-73.

25 van Zoelen MAD, Laterre P-F, van Veen SQ, et al. Systemic and local high mobility group box 1 concentrations during severe infection. Crit Care Med 2007;35:2799-804.

26 Gibot S, Massin F, Cravoisy A, et al. High-mobility group box 1 protein plasma concentrations during septic shock. Intensive Care Med 2007;33:1347-53

27 Winter N, Meyer A, Richter A, et al. Elevated levels of HMGB1 in cancerous and inflammatory effusions. Anticancer Res 2009;29:5013-7.

28 Tang K-T, Hsieh T-Y, Chao Y-H, et al. Plasma levels of high-mobility group box 1 and soluble receptor for advanced glycation end products in primary antiphospholipid antibody syndrome patients. PLoS One 2017;12:e0178404

29 Sim YS, Kim DG, Shin TR. The diagnostic utility and tendency of the soluble receptor for advanced glycation end products (sRAGE) in exudative pleural effusion. J Thorac Dis 2016;8:1731-7.

30 Gao Z-W, Wang X, Zhang H-Z, et al. The roles of adenosine deaminase in autoimmune diseases. Autoimmun Rev 2021;20:102709.

31 Saghiri R, Ghashghai N, Movaseghi S, et al. Serum adenosine deaminase activity in patients with systemic lupus erythematosus: a study based on ADA1 and ADA2 isoenzymes pattern. Rheumatol Int 2012;32:1633-8.

32 Gao Z-W, Zhao G-H, Zhang Z, et al. Serum adenosine deaminase activity is increased in systemic lupus erythematosus patients and correlated with disease activity. Immunol Res 2018;66:299-304.

33 Hoffmann JP, Kolls JK, McCombs JE. Regulation and function of ILC3s in pulmonary infections. Front Immunol 2021;12:672523.

34 Wang DY, Yang PC, Yu WL, et al. Serial antinuclear antibodies titre in pleural and pericardial fluid. Eur Respir J 2000;15:1106-10.

35 Toworakul C, Kasitanon N, Sukitawut W, et al. Usefulness of pleura effusion antinuclear antibodies in the diagnosis of lupus pleuritis. Lupus 2011;20:1042-6. 
36 Tedeschi SK, Johnson SR, Boumpas D, et al. Developing and refining new candidate criteria for systemic lupus erythematosus classification: an international collaboration. Arthritis Care Res 2018;70:571-81.

37 Willems P, De Langhe E, Westhovens R, et al. Antinuclear antibody as entry criterion for classification of systemic lupus erythematosus: pitfalls and opportunities. Ann Rheum Dis 2019;78:e76.

38 Glovsky MM, Louie JS, Pitts WH, et al. Reduction of pleural fluid complement activity in patients with systemic lupus erythematosus and rheumatoid arthritis. Clin Immunol Immunopathol 1976;6:31-41.
39 Hunder GG, McDuffie FC, Hepper NG. Pleural fluid complement in systemic lupus erythematosus and rheumatoid arthritis. Ann Intern Med 1972;76:357-63.

40 Hunder GG, McDuffie FC, Huston KA, et al. Pleural fluid complement, complement conversion, and immune complexes in immunologic and nonimmunologic diseases. J Lab Clin Med 1977;90:971-80.

41 Salomaa ER, Viander M, Saaresranta T, et al. Complement components and their activation products in pleural fluid. Chest 1998;114:723-30.

42 Truedsson L, Bengtsson AA, Sturfelt G. Complement deficiencies and systemic lupus erythematosus. Autoimmunity 2007;40:560-6. 